\title{
Magnetism in spin models for depleted honeycomb-lattice iridates: Spin-glass order towards percolation
}

\author{
Eric C. Andrade \\ Institut für Theoretische Physik, Technische Universität Dresden, 01062 Dresden, Germany \\ and Instituto de Física Teórica, Universidade Estadual Paulista, Rua Dr. Bento Teobaldo Ferraz 271, \\ Bl. II, 01140-070 São Paulo, SP, Brazil \\ Matthias Vojta \\ Institut für Theoretische Physik, Technische Universität Dresden, 01062 Dresden, Germany
}

(Received 24 September 2013; revised manuscript received 16 October 2014; published 7 November 2014)

\begin{abstract}
Iridates are characterized by a fascinating interplay of spin-orbit and electron-electron interactions. The honeycomb-lattice materials $A_{2} \mathrm{IrO}_{3}(A=\mathrm{Na}, \mathrm{Li})$ have been proposed to realize pseudospin-1/2 Mott insulating states with strongly anisotropic exchange interactions, described by the Heisenberg-Kitaev model, but other scenarios involving longer-range exchange interactions or more delocalized electrons have been put forward as well. Here we study the influence of nonmagnetic doping, i.e., depleted moments, on the magnetic properties of experimentally relevant variants of the Heisenberg-Kitaev and Heisenberg $J_{1}-J_{2}-J_{3}$ models. We generically find that the zigzag order of the clean system is replaced, upon doping, by a spin-glass state with short-ranged zigzag correlations. We determine the spin-glass temperature as a function of the doping level and show that this quantity allows one to assess the importance of longer-range exchange interactions when the doping is driven across the site-percolation threshold of the honeycomb lattice.
\end{abstract}

DOI: 10.1103/PhysRevB.90.205112

PACS number(s): 75.10.Nr, 74.72.-h, 75.10.Jm, 75.50.Ee

\section{INTRODUCTION}

The interplay of strong spin-orbit coupling and electronic correlations is at the heart of many recent developments in condensed-matter physics, involving, e.g., correlated topological insulators, fractional Chern insulators, and spin-orbit Mott insulators [1-5]. On the materials side, oxides with partially filled $5 d$ shells, such as iridates and osmates, are considered promising candidates in order to realize the theoretically proposed phenomena.

In this context, the insulating iridates $A_{2} \mathrm{IrO}_{3}(A=\mathrm{Na}, \mathrm{Li})$ have attracted enormous attention over the past few years [6-11]. In these materials, the $\operatorname{Ir}^{4+}$ ions are arranged in a layered honeycomb-lattice structure. Due to the combined effect of strong spin-orbit coupling and Coulomb interactions, the Ir $5 d^{5}$ states, with one hole in the $t_{2 g}$ manifold, have been proposed to realize $J_{\text {eff }}=1 / 2$ spin-orbit Mott insulators $[12,13]$, similar to other layered iridates [4,5]. Furthermore, Ref. [14] suggested that the magnetism of the $J_{\text {eff }}=1 / 2$ moments is dominated by strongly spin-anisotropic compass interactions, which by itself lead to the spin-liquid model on the honeycomb lattice proposed by Kitaev [15]. Supplemented by additional spin-isotropic Heisenberg interactions, the resulting Heisenberg-Kitaev (HK) model has been shown to host both spin-liquid and conventionally ordered phases [14,16-21].

Experimentally, both $\mathrm{Na}_{2} \mathrm{IrO}_{3}$ and $\mathrm{Li}_{2} \mathrm{IrO}_{3}$ have been found to undergo a magnetic ordering transition at $T_{\mathrm{N}} \simeq 15 \mathrm{~K}$ [6-8]. In $\mathrm{Na}_{2} \mathrm{IrO}_{3}$ the low-temperature spin configuration has been identified as collinear "zigzag" order $[9,10]$, with ferromagnetic zigzag chains arranged antiferromagnetically in the honeycomb plane. This state is indeed a ground state of the HK model, where it results from a competition of antiferromagnetic Kitaev and ferromagnetic Heisenberg interactions [19]. Alternatively, Heisenberg and HK models with longer-range interactions have been considered: Specifi- cally, a Heisenberg $J_{1}-J_{2}-J_{3}$ model with sizable second- and third-neighbor coupling has been found to describe the available data as well $[9,22]$. Finally, a more itinerant scenario in terms of molecular orbitals has also been proposed [23], although a detailed description of the magnetic properties in this model is lacking to date.

In this paper, we propose magnetic depletion, i.e., the random substitution of magnetic $\mathrm{Ir}^{4+}$ by nonmagnetic ions, as a powerful tool to study the magnetism of $A_{2} \mathrm{IrO}_{3}$ and to discriminate between the various proposed scenarios for magnetism. A key insight is that, within local-moment models, depletion will inevitably turn the zigzag ordered state into a spin (or spin-orbit) glass: Both the HK and $J_{1}-J_{2}-J_{3}$ models are frustrated, and the combination of disorder and frustration generically causes spin-glass behavior [24]. We calculate the freezing temperature $T_{\mathrm{g}}(x)$ as a function of doping level $x$ and show that its behavior across the site-percolation threshold, $x_{p}=30.3 \%$, strongly differs between the HK and $J_{1}-J_{2}-J_{3}$ models (Fig. 1).

\section{MODELS}

We focus on two models which have been considered to describe the zigzag ordered state of $\mathrm{Na}_{2} \mathrm{IrO}_{3}$. The first is the HK model,

$$
\mathcal{H}=J \sum_{\langle i j\rangle} \vec{S}_{i} \cdot \vec{S}_{j}+2 K \sum_{\langle i j\rangle_{\gamma}} S_{i}^{\gamma} S_{j}^{\gamma}
$$

and the second is the $J_{1}-J_{2}-J_{3}$ model,

$$
\mathcal{H}=J_{1} \sum_{\langle i j\rangle} \vec{S}_{i} \cdot \vec{S}_{j}+J_{2} \sum_{\langle\langle i k\rangle\rangle} \vec{S}_{i} \cdot \vec{S}_{k}+J_{3} \sum_{\langle\langle\langle i l\rangle\rangle\rangle} \vec{S}_{i} \cdot \vec{S}_{l} .
$$

Here, the sums run over pairs of nearest-, second-, and third-neighbor sites, respectively, while $\gamma=x, y, z$ in Eq. (1) 

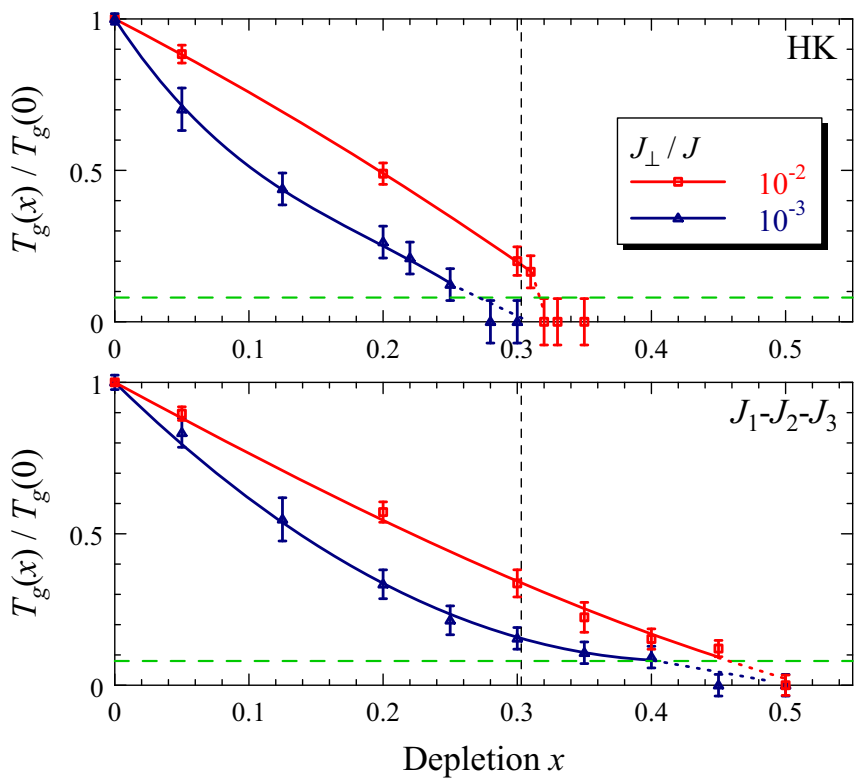

FIG. 1. (Color online) Freezing temperature extracted from our Monte Carlo (MC) simulations, shown as $T_{\mathrm{g}}(x) / T_{\mathrm{g}}(x=0)$ as a function of doping level $x$, for the HK (top) and $J_{1}-J_{2}-J_{3}$ (bottom) models for two different values of the interlayer coupling $J_{\perp} / J$. The vertical dashed line locates the two-dimensional (2D) percolation threshold $x_{p}$; the horizontal dashed line marks temperatures below which we are unable to reach equilibrium in our MC simulations. Solid lines are polynomial fits through the data; dotted lines are extrapolations.

labels the three different links for each spin in a honeycomb lattice. The parameter regimes of interest are defined through the presence of zigzag magnetic order as realized in $\mathrm{Na}_{2} \mathrm{IrO}_{3}$ $[9,10]$. The HK model's couplings may be parametrized as $J=A \cos \phi$ and $K=A \sin \phi$, where $A$ is an overall energy scale. Its full phase diagram was first mapped out in Ref. [19], with the zigzag phase occurring for $0.51 \pi<\phi<0.90 \pi$; in the following we choose $\phi=0.62 \pi$. For the $J_{1}-J_{2}-J_{3}$ model, sizable $J_{2}$ and $J_{3}$ are required in order to have a zigzag magnetic ground state [25-27]. Following Ref. [9], we choose $J_{2}=0.8 J_{1}$ and $J_{3}=0.9 J_{1}$.

As will become clear below, the magnetic properties of the depleted $\mathrm{HK}$ and $J_{1}-J_{2}-J_{3}$ models depend sensitively on the presence of a magnetic coupling between the layers. For $A_{2} \mathrm{IrO}_{3}$, no quantitative information on such coupling is available at present; it is often assumed to be small due to the A-B-type stacking of the honeycomb layers. Here we will account for the three-dimensional (3D) character by considering a layered model with A-A stacking and a small vertical (unfrustrated) Heisenberg coupling $J_{\perp}$; in application to $A_{2} \mathrm{IrO}_{3}$ this is to be understood as an effective coupling between second-neighbor layers.

\section{MONTE CARLO SIMULATIONS}

We study the models (1) and (2) using classical Monte Carlo (MC) simulations for unit-length spins on lattices of size $L \times L \times L_{z}$, typically with $L_{z}=L / 2$ and periodic boundary conditions. The honeycomb layers are spanned by the primitive lattice vectors $\vec{a}_{1(2)}=(3 / 2, \pm \sqrt{3} / 2)$, with each unit cell containing two sites. Depletion is simulated by randomly removing a fraction $x$ of spins, with $x$ varying between $5 \%$ and $50 \%$, with the total number of spins $N_{s}=(1-x) 2 L^{2} L_{z}$. We perform equilibrium MC simulations using single-site updates with a combination of the heat-bath and microcanonical (or overrelaxation) methods, with typically $10^{6} \mathrm{MC}$ steps per spin, and combine this with the parallel-tempering algorithm [28,29]. Disorder averages are taken over $N_{\mathrm{rl}}$ samples, with $N_{\mathrm{rl}}$ ranging from 1000 for $L=8$ to $N_{\mathrm{rl}}=50$ for $L=20$. Below we quote energies in units of $J \equiv J_{1}$, the nearest-neighbor Heisenberg exchange.

We extract the ordering (or freezing) temperature $T_{\mathrm{g}}$ from the crossing points of $\xi(T) / L$ for different $L$, according to the scaling law $\xi / L=f\left(L^{1 / \nu}\left(T-T_{\mathrm{g}}\right)\right)$, where $\xi$ is a correlation length, $f(x)$ a scaling function, and $v$ the correlation length exponent. This procedure is especially suitable to detect spinglass freezing, as shown in previous studies of the 3D EdwardsAnderson model [30-32]. The main source of numerical error in $T_{\mathrm{g}}$ is from the $L \rightarrow \infty$ extrapolation of the crossing-point location required for small $L$.

The magnetic correlation length $\xi_{S}$ is calculated from a fit of the static magnetic structure factor $S(\vec{q})$ close to the ordering wave vector $\vec{Q}$ [the three independent $\vec{Q}$ vectors corresponding to the zigzag order are $\left(\vec{b}_{1}+\vec{b}_{2}\right) / 2$, $\vec{b}_{1} / 2$, and $\vec{b}_{2} / 2$, where $\vec{b}_{1(2)}=2 \pi(1 / 3, \pm 1 / \sqrt{3})$ are the reciprocal lattice vectors]. Analogously, the spin-glass correlation length $\xi_{\mathrm{SG}}$ is obtained from the spin-glass susceptibility $\chi_{\mathrm{SG}}(\vec{q})=N_{s} \sum_{\alpha, \beta}\left[\left\langle\left|q^{\alpha, \beta}(\vec{q})\right|^{2}\right\rangle\right]_{\mathrm{avg}}$, where $q^{\alpha, \beta}(\vec{q})=$ $N_{s}^{-1} \sum_{i} S_{i}^{\alpha(1)} S_{i}^{\beta(2)} \exp \left(i \vec{q} \cdot \vec{r}_{i}\right)$ is the spin-glass order parameter. Here $\alpha$ and $\beta$ are spin components, superscripts $(1,2)$ denote identical copies of the system ("replicas") containing the disorder configuration, $\langle\cdots\rangle$ denotes the MC average, and $[\cdots]_{\text {avg }}$ the average over disorder.

\section{CLEAN HK MODEL}

The 2D disorder-free HK model has been studied by various numerical methods [14,17,19-21]. A comparison of phase diagrams shows that the classical-spin HK model reproduces [21] all phases of the spin-1/2 model except for the quantum spin liquid [19], with $T=0$ phase-boundary locations in reasonable agreement between quantum and classical models. The results in Refs. [20,21] also indicate two thermal transitions upon cooling to any of the ordered low- $T$ phases. The system enters a critical phase at $T_{\mathrm{u}}$, with power-law spin correlations, and a state with true long-range order is reached only below $T_{1}<T_{\mathrm{u}}$. This behavior parallels that of a 2D six-state clock model [33], as suggested by the sixfold degeneracy of the ordered states in the HK model.

For selected values of $\phi$, we have verified that our MC simulations, applied to the $2 \mathrm{D} \mathrm{HK}$ model $\left(J_{\perp}=0\right)$, reproduce the results of Ref. [21]. In particular, the specific heat [Fig. 2(a)] shows a broad peak far above both $T_{\mathrm{u}}$ and $T_{1}$ while the singularity at the transitions is weak - this reflects the presence of strong fluctuations in the 2D system. Nevertheless, there is a well-defined crossing point in $\xi_{\mathrm{S}} / L$ at $T_{1}$ where long-range order sets in [Fig. 2(c)]. 

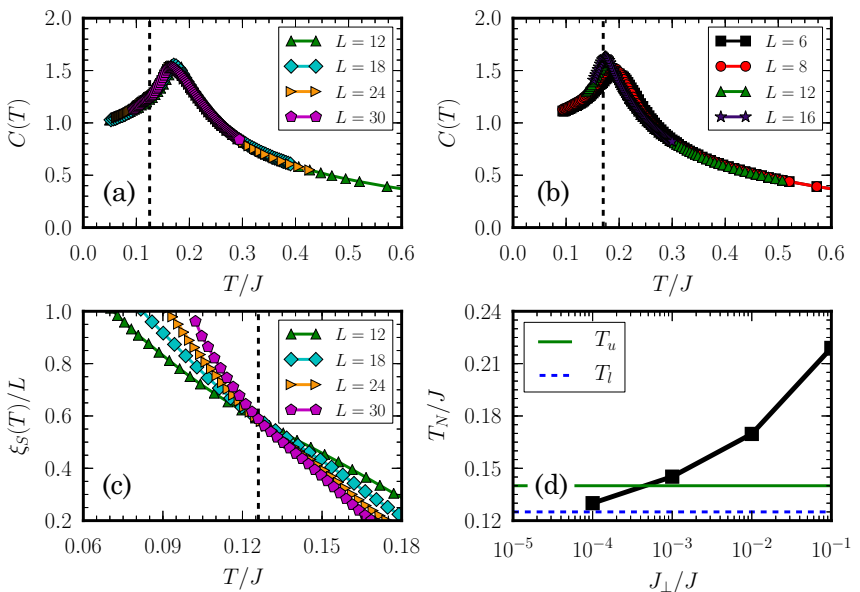

FIG. 2. (Color online) MC results for the ordering into the zigzag state of the clean HK model for several system sizes $L$. (a), (c) 2D. (b), (d) 3D. (a), (b) Specific heat as a function of the temperature $T$, in (b) with $J_{\perp} / J=10^{-2}$. (c) $\xi_{\mathrm{S}} / L$ as a function of $T$. The vertical dashed line indicates $T_{1}$. (d) $T_{\mathrm{N}}\left(J_{\perp} / J\right)$, also showing $T_{\mathrm{u}}$ and $T_{1}$ of the 2D HK model; the value of $T_{\mathrm{u}}$ was extracted from Ref. [21].

We have then switched on the interlayer coupling $J_{\perp}$ and monitored the evolution of the transition temperature [Fig. 2(d)]. As expected on general grounds, the critical intermediate phase of the 2D system disappears for finite $J_{\perp}$, such that there is only a single thermal phase transition at $T_{\mathrm{N}}$, which now displays a pronounced specific-heat singularity [Fig. 2(b)]. For $J_{\perp} / J \gtrsim 10^{-3}, T_{\mathrm{N}}$ is larger than both $T_{1}$ and $T_{\mathrm{u}}$ of the 2D system, and our data are compatible with $T_{\mathrm{N}} \rightarrow T_{1}$ as $J_{\perp} \rightarrow 0$, although finite-size effects hamper an accurate determination of $T_{\mathrm{N}}$ for $J_{\perp} / J<10^{-4}$ [Fig. 2(d)].

Clean $J_{1}-J_{2}-J_{3}$ model. We have also performed corresponding simulations for the $J_{1}-J_{2}-J_{3}$ model. Here, $T_{N} \rightarrow 0$ for $J_{\perp} \rightarrow 0$ due to the assumed continuous spin symmetry. For $J_{\perp} / J=10^{-2}\left[10^{-3}\right]$ we have $T_{\mathrm{N}} / J=0.446(3)[0.42(1)]$.

\section{MAGNETIC DEPLETION}

We now describe our central results, obtained for the depleted $\mathrm{HK}$ and $J_{1}-J_{2}-J_{3}$ models, with a concentration $x$ of randomly placed vacancies. Since both models are frustrated, the introduction of vacancies generate local noncollinearities in the spin order [34], which ultimately leads to spin-glass behavior [24].

We have first studied the 2D case $\left(J_{\perp}=0\right)$ and found-in both models and for any $x \geqslant 5 \%$-indications of neither conventional nor spin-glass order at finite temperature. This is expected: Conventional order is suppressed, due to the combination of disorder and frustration, in favor of spin-glass magnetism. However, the glass temperature is strictly zero in two dimensions $[35,36]$, even in the case of Ising symmetry.

For finite interlayer coupling the situation changes, with sample results shown in Fig. 3. While conventional long-range order is absent for any $x \geqslant 5 \%$, spin-glass order emerges instead at low $T$. The latter is signified by a well-defined common crossing point in $\xi_{\mathrm{SG}} / L$ and a corresponding scaling [Figs. 3(c) and 3(d)] [37]. In contrast, existing crossing points of $\xi_{\mathrm{S}} / L$ display a systematic downward shift with increasing $L$,
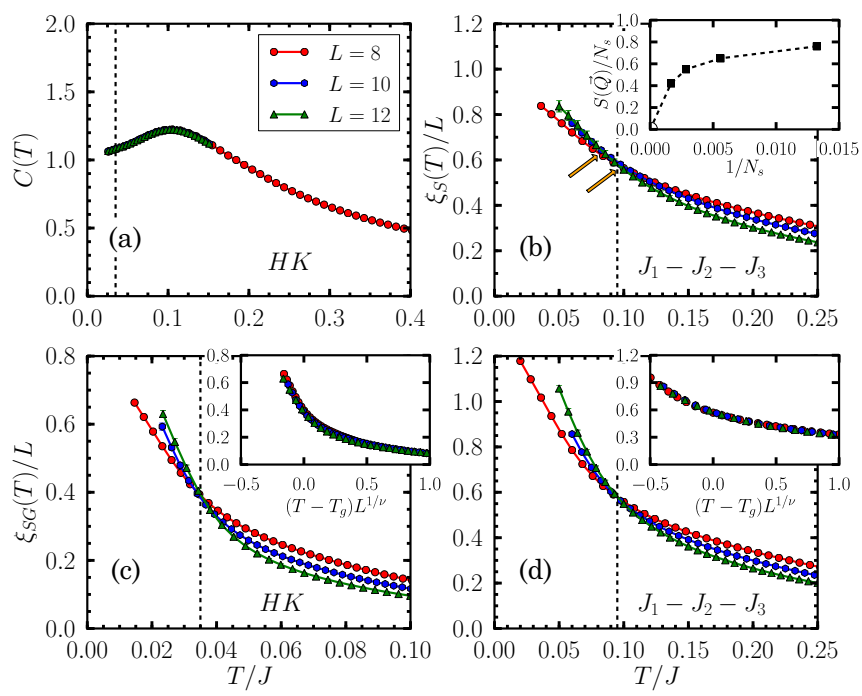

FIG. 3. (Color online) $\mathrm{MC}$ results for the ordering into the spinglass state for $J_{\perp} / J=10^{-2}$. (a), (c) HK model at $x=30 \%$. (b), (d) $J_{1}-J_{2}-J_{3}$ model at $x=35 \%$. (a) Specific heat as a function of the temperature $T$. (b) $\xi_{\mathrm{S}} / L$ as a function of $T$. The arrows indicate the crossing points of the curves for different pairs of $L$, displaying a clear downward trend with increasing $L$. Inset: Bragg intensity $S\left(\vec{Q} / N_{s}\right)$ extrapolated to $T \rightarrow 0$ as a function of $1 / N_{s}$. (c), (d) $\xi_{\mathrm{SG}} / L$ as a function of $T$. Inset: Scaling plot with $\xi_{\mathrm{SG}} / L$ as a function of $\left(T-T_{\mathrm{g}}\right) L^{1 / \nu}$. The vertical dashed lines indicate the glass temperature $T_{\mathrm{g}}$, as determined in (c) and (d).

indicative of short-range zigzag spin correlations [Fig. 3(b)]. We note that we do not reach the limit $L \gg \xi_{\mathrm{S}}(T=0)$ where crossing points would be absent entirely.

Short-range magnetic order also manifests itself in the specific heat [Fig. 3(a)]. The peak in $C(T)$ is broad and occurs at a temperature considerably larger than the freezing temperature (here $T_{\text {peak }} \approx 2 T_{\mathrm{g}}$ ), indicating that this short-range order builds up at temperatures considerably higher than $T_{\mathrm{g}}$. We stress that this behavior is a hallmark of glassy systems $[29,36]$, and it is, in principle, disconnected from the nontrivial behavior of the 2D disorder-free HK model [20] [Fig. 2(a)].

To account for the possibility of different (non-zigzag) dilution-induced magnetic ground states, we monitored $S(\vec{q})$ in the reciprocal space, but (within our resolution) we detected peaks only at the $\vec{Q}$ vectors corresponding to the zigzag order. However, these peaks grow slower than the system size [Fig. 3(b)], again indicating static short-range order with a vanishing magnetic order parameter.

\section{SMALL DOPING AND GLASSINESS}

In both the Heisenberg-Kitaev and $J_{1}-J_{2}-J_{3}$ models, we find a single vacancy to produce anticollinear states [34]. Multiple vacancies have a somewhat different effect in the two models: In the Heisenberg-Kitaev case, the vacancies locally select specific stripe orientations due to spin-orbit coupling [38], causing domains with different stripe orientations to coexist. In the $J_{1}-J_{2}-J_{3}$ model, instead, the effect of long-range distortions of the spin pattern is more prominent, due to the presence of gapless bulk modes. Remarkably, for the vacancy concentrations of interest, $x \geqslant 20 \%$, the spin configurations 


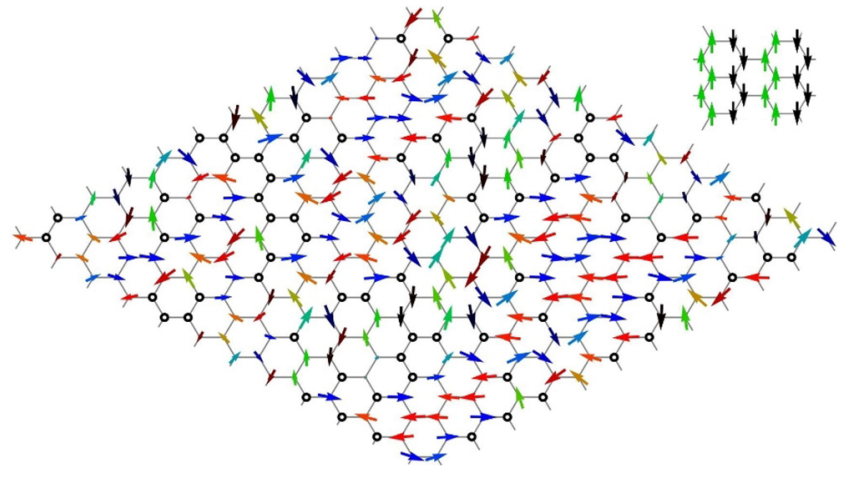

FIG. 4. (Color online) Sample ground-state spin configuration of the classical HK model at $x=20 \%$ depletion, with one $L=12$ layer shown. The arrows denote the $x$ and $z$ components of the $\vec{S}_{i}$; the circles indicate the vacancy positions. The arrow lengths indicate the weight of the projection onto the $x-z$ plane and the colors the in-plane orientation. Short-range zigzag order with glassy domain formation is visible. Inset: Ideal zigzag order with the spins aligned along $S_{i}^{z}$.

we observe in both models are virtually indistinguishable and are characterized by a short-range domain structure (Fig. 4).

Based on our MC data, we are unable to decide whether long-range order is destroyed in favor of spin-glass order at infinitesimal $x$ or at a finite critical $x_{c}$ (with $x_{c}<5 \%$ ). We leave a more detailed characterization of the small-doping behavior for future work.

\section{ORDERING TEMPERATURE AND PERCOLATION}

An easily accessible quantity is the ordering (or freezing) temperature $T_{\mathrm{g}}$ as function of $x$. While one generally expects that $T_{\mathrm{g}}$ decreases with increasing $x$, the behavior at large $x$ contains information on the nature of the magnetic couplings: For a layered local-moment system with nearest-neighbor couplings, $T_{\mathrm{g}}$ will diminish near the threshold $x_{p}$ for $2 \mathrm{D}$ site percolation, because for $x>x_{p}$ the layers fragment into disconnected spin clusters, and for Heisenberg symmetry $T_{\mathrm{g}}(x) / T_{\mathrm{g}}(x=0)$ will vanish as $x \rightarrow x_{p}$ in the limit of small interlayer coupling. In contrast, in systems with longer-range magnetic couplings, $T_{\mathrm{g}}$ will stay finite across $x_{p}$ [39]. The parent compounds of cuprate superconductors exemplify this physics: The Néel temperature in $\mathrm{Zn}$-doped $\mathrm{La}_{2} \mathrm{CuO}_{4}$ vanishes essentially at the square-lattice percolation threshold of $x_{p}=40.5 \%$ [40]—-this proves that the cuprate magnetism is dominated by nearest-neighbor coupling.

Our results for $T_{\mathrm{g}}(x)$ are shown in Fig. 1. As expected from the above discussion, $T_{\mathrm{g}}$ of the nearest-neighbor $\mathrm{HK}$ model rapidly drops towards the honeycomb lattice $x_{p}=30.3 \%$ and becomes smaller than our lowest simulation temperature $(J / 80)$ for $x \gtrsim 32 \%$ for $J_{\perp} / J=10^{-2}$. (Note that, due to the finite $J_{\perp}, T_{\mathrm{g}}$ is expected to be nonvanishing up to the 3D percolation threshold, however, it is undetectably small for $x \gtrsim 32 \%$.) For smaller interlayer coupling this apparent vanishing of $T_{\mathrm{g}}(x)$ appears at even smaller $x$.

In contrast, $T_{\mathrm{g}}$ of the $J_{1}-J_{2}-J_{3}$ model continues its approximately linear variation with $x$ across $x_{p}$ and extrapolates to our lowest simulation temperature at a much larger doping level of $x \approx 50 \%$ [41]. For both models, $T_{\mathrm{g}}(x) / T_{\mathrm{g}}(x=0)$ diminishes with decreasing $J_{\perp} / J$, and small $J_{\perp} / J$ induce a curvature in $T_{\mathrm{g}}(x)$, which is particularly pronounced at small $x$.

\section{SUMMARY}

We have studied the magnetism of local-moment models for $A_{2} \mathrm{IrO}_{3}$ under magnetic depletion. A spin-orbit glass, with zigzag short-range order, emerges generically from the combination of strong spin-orbit coupling, frustration, and disorder. We have determined the glass (or freezing) temperature $T_{\mathrm{g}}$ as a function of the doping level $x$, which at large doping differs qualitatively between the $\mathrm{HK}$ and $J_{1}-J_{2}-J_{3}$ models [Fig. 1(a)].

We thus propose to employ magnetic depletion, using dopants with magnetically inert $d$ shells, as a tool to assess the importance of longer-range magnetic couplings in the $A_{2} \mathrm{IrO}_{3}$ compounds: If the experimental $T_{\mathrm{g}}$ were found to vanish near $x_{p}$, this would strongly hint [42] at short-range HK physics being realized in $A_{2} \mathrm{IrO}_{3}$, as originally proposed in Refs. [14,19]. Conversely, the absence of such vanishing would imply significant longer-range interactions.

Note added. Very recent experiments [43], using nonmagnetic Ti dopants substituting for Ir, show significant differences between depleted $\mathrm{Na}_{2} \mathrm{IrO}_{3}$ and $\mathrm{Li}_{2} \mathrm{IrO}_{3}$ across the percolation threshold.

\section{ACKNOWLEDGMENTS}

We thank J. van den Brink, P. Gegenwart, P. Horsch, G. Khaliullin, and A. Rosch for discussions. The computations were partially performed at the Center for Information Services and High Performance Computing (ZIH) at TU Dresden. This research was supported by the DFG through FOR 960 and GRK 1621 as well as by the Helmholtz association through VI521. E.C.A. was also partially supported by FAPESP (Brazil).
[1] D. A. Pesin and L. Balents, Nat. Phys. 6, 376 (2010).

[2] M. Hohenadler and F. F. Assaad, J. Phys.: Condens. Matter 25, 143201 (2013).

[3] E. J. Bergholtz and Z. Liu, Int. J. Mod. Phys. B 27, 1330017 (2013).

[4] B. J. Kim, H. Jin, S. J. Moon, J.-Y. Kim, B.-G. Park, C. S. Leem, J. Yu, T. W. Noh, C. Kim, S.-J. Oh, J.-H. Park, V. Durairaj, G. Cao, and E. Rotenberg, Phys. Rev. Lett. 101, 076402 (2008).
[5] B. J. Kim, H. Ohsumi, T. Komesu, S. Sakai, T. Morita, H. Takagi, and T. Arima, Science 323, 1329 (2009).

[6] Y. Singh and P. Gegenwart, Phys. Rev. B 82, 064412 (2010).

[7] X. Liu, T. Berlijn, W.-G. Yin, W. Ku, A. Tsvelik, Y.-J. Kim, H. Gretarsson, Y. Singh, P. Gegenwart, and J. P. Hill, Phys. Rev. B 83, 220403 (2011).

[8] Y. Singh, S. Manni, J. Reuther, T. Berlijn, R. Thomale, W. Ku, S. Trebst, and P. Gegenwart, Phys. Rev. Lett. 108, 127203 (2012). 
[9] S. K. Choi, R. Coldea, A. N. Kolmogorov, T. Lancaster, I. I. Mazin, S. J. Blundell, P. G. Radaelli, Y. Singh, P. Gegenwart, K. R. Choi, S.-W. Cheong, P. J. Baker, C. Stock, and J. Taylor, Phys. Rev. Lett. 108, 127204 (2012).

[10] F. Ye, S. Chi, H. Cao, B. C. Chakoumakos, J. A. Fernandez-Baca, R. Custelcean, T. F. Qi, O. B. Korneta, and G. Cao, Phys. Rev. B 85, 180403 (2012).

[11] R. Comin, G. Levy, B. Ludbrook, Z.-H. Zhu, C. N. Veenstra, J. A. Rosen, Y. Singh, P. Gegenwart, D. Stricker, J. N. Hancock, D. van der Marel, I. S. Elfimov, and A. Damascelli, Phys. Rev. Lett. 109, 266406 (2012).

[12] G. Jackeli and G. Khaliullin, Phys. Rev. Lett. 102, 017205 (2009).

[13] A. Shitade, H. Katsura, J. Kuneš, X.-L. Qi, S.-C. Zhang, and N. Nagaosa, Phys. Rev. Lett. 102, 256403 (2009).

[14] J. Chaloupka, G. Jackeli, and G. Khaliullin, Phys. Rev. Lett. 105, 027204 (2010).

[15] A. Kitaev, Ann. Phys. (NY) 321, 2 (2006).

[16] H.-C. Jiang, Z.-C. Gu, X.-L. Qi, and S. Trebst, Phys. Rev. B 83, 245104 (2011).

[17] J. Reuther, R. Thomale, and S. Trebst, Phys. Rev. B 84, 100406 (2011).

[18] S. Bhattacharjee, S.-S. Lee, and Y. B. Kim, New J. Phys. 14, 073015 (2012).

[19] J. Chaloupka, G. Jackeli, and G. Khaliullin, Phys. Rev. Lett. 110, 097204 (2013).

[20] C. C. Price and N. B. Perkins, Phys. Rev. Lett. 109, 187201 (2012).

[21] C. C. Price and N. B. Perkins, Phys. Rev. B 88, 024410 (2013).

[22] I. Kimchi and Y.-Z. You, Phys. Rev. B 84, 180407 (2011).

[23] I. I. Mazin, H. O. Jeschke, K. Foyevtsova, R. Valenti, and D. I. Khomskii, Phys. Rev. Lett. 109, 197201 (2012).

[24] J. Villain, Z. Phys. B 33, 31 (1979).

[25] E. Rastelli, A. Tassi, and L. Reatto, Physica B+C 97, 1 (1979).
[26] J. Fouet, P. Sindzingre, and C. Lhuillier, Eur. Phys. J. B 20, 241 (2001).

[27] P. H. Y. Li, R. F. Bishop, D. J. J. Farnell, and C. E. Campbell, Phys. Rev. B 86, 144404 (2012).

[28] For further details, see Ref. [29].

[29] E. C. Andrade and M. Vojta, Europhys. Lett. 97, 37007 (2012).

[30] L. W. Lee and A. P. Young, Phys. Rev. Lett. 90, 227203 (2003).

[31] I. Campos, M. Cotallo-Aban, V. Martin-Mayor, S. Perez-Gaviro, and A. Tarancon, Phys. Rev. Lett. 97, 217204 (2006).

[32] D. X. Viet and H. Kawamura, Phys. Rev. Lett. 102, 027202 (2009).

[33] J. V. Jose, L. P. Kadanoff, S. Kirkpatrick, and D. R. Nelson, Phys. Rev. B 16, 1217 (1977).

[34] C. L. Henley, Phys. Rev. Lett. 62, 2056 (1989).

[35] A. J. Bray and M. A. Moore, J. Phys. C 17, L463 (1984).

[36] K. H. Fischer and J. A. Hertz, Spin Glasses (Cambridge University Press, Cambridge, UK, 1991).

[37] For both models, we estimate the same correlation length exponent $v \approx 1.05$. Due to our modest system sizes, we do not pursue a complete characterization of the critical exponents.

[38] F. Trousselet, G. Khaliullin, and P. Horsch, Phys. Rev. B 84, 054409 (2011).

[39] A lattice with further-neighbor links has a larger site-percolation threshold than the one with nearest-neighbor links only.

[40] O. P. Vajk, P. K. Mang, M. Greven, P. M. Gehring, and J. W. Lynn, Science 295, 1691 (2002).

[41] A honeycomb lattice with only $J_{2}$ couplings nonzero corresponds to two decoupled triangular lattices; the site-percolation threshold of a triangular lattice is $x=50 \%$.

[42] In more itinerant scenarios of $A_{2} \mathrm{IrO}_{3}$ magnetism, such as in Ref. [23], site percolation is not expected to drastically influence $T_{\mathrm{g}}(x)$.

[43] S. Manni, Y. Tokiwa, and P. Gegenwart, Phys. Rev. B 89, 241102(R) (2014). 